Chirurg 2015 $\cdot 86: 1005-1006$

DOI 10.1007/s00104-015-0088-7

Online publiziert: 22. September 2015

(c) Springer-Verlag Berlin Heidelberg 2015

\section{U. Settmacher}

Klinik für Allgemein-, Viszeral- und Gefäßchirurgie, Universitätsklinikum Jena, Jena, Deutschland

\title{
Management von Komplikationen nach Operationen am oberen Gastrointestinaltrakt
}

Ein großer Teil viszeralchirurgischer Operationen sind Operationen am oberen Gastrointestinaltrakt, d. h. am Ösophagus, Magen und Duodenum. Hier hat sich das Spektrum der Indikationen bereits seit längerer Zeit hauptsächlich zur Chirurgie von Malignomen gewendet. Minimal-invasive Techniken haben Einzug in die Behandlung gefunden. So werden Frühbefunde langfristig sehr erfolgreich endoskopisch abgetragen. Ebenso, wenn auch etwas verhaltener im Vergleich zum unteren Gastrointestinaltrakt, ist auch am oberen Gastrointestinaltrakt die laparoskopische inklusive die roboterassistierte Chirurgie in Entwicklung und Verbreitung. Um die Ergebnisse der Malignomchirurgie bei fortgeschrittenen Stadien zu verbessern, wurden neoadjuvante Protokolle der Strahlen- und oder Chemotherapie eingeführt.

Wir haben uns deshalb entschieden eine Diskussion des Managements von Komplikationen nach Interventionen am oberen Gastrointestinaltrakt an vier Unterthemen zu führen.

Begonnen wird dem Beitrag von Y. Dittmar et al., die das Management von Komplikationen bei endoskopischen Interventionen darstellen. Diese Untersuchungs- und Behandlungsverfahren sind sicher und komplikationsarm. In vielen Fällen kann die chirurgische Behandlung durch das breite Repertoire interventioneller Therapieoptionen zwar vermieden werden, dennoch sollte die schwere endoskopische Komplikation von Anfang an durch den Chirurgen begleitet werden. Auch bei diesen Komplikationen sind eine zeitnahe Diagnosestellung und ein strukturiertes Management die wich- tigsten Voraussetzungen für eine effiziente Behandlung. Man kann hier eine Einteilung in prä-, peri- und postinterventionelle Komplikationen didaktisch abarbeiten. Blutungen und Perforationen sind dabei die periinterventionellen Ereignisse mit der größten Problematik. Gerade für diese Komplikationen gibt es eine Reihe endoskopischer Therapiemöglichkeiten, die ein konventionell chirurgisches Intervenieren heute oft nicht nötig werden lassen.

\section{》) Problematisch ist die Kardiotoxizität insbesondere von Fluoropyrimidinen}

Der zweite Beitrag dieses Leitthemas von I. Gockel et al. beschäftigt sich mit dem Management von Komplikationen unter und nach neoadjuvanter Therapie. Auch hier spielen eine sachgerechte Prophylaxe und ein professionelles Komplikationsmanagement durch das Vermeiden bzw. eine ausreichende Kontrolle der Komplikation eine entscheidende Rolle. Empfohlen wird, dass am Behandlungszentrum rund um die Uhr eine Notfalleinrichtung geöffnet sein soll, die alle Notfallmaßnahmen in die Wege leiten kann. Komplikationen können durch den Tumor selbst oder durch die Therapie hervorgerufen werden. Diese umfassen Blutungen, Fisteln, Perforationen und Stenosen. Moderne endoskopische Techniken stellen hier die Therapie der Wahl dar. Nebenwirkungen der neoadjuvanten Therapie wie hämatologische Toxizität, Übelkeit und Erbrechen, Neurotoxizität, Hauttoxizität werden konservativ therapiert. Pro- blematisch ist die Kardiotoxizität, da sie insbesondere nach Fluoropyrimidinen die Operabilität infrage stellen kann, sodass mitunter die Therapie beendet werden muss. Ein Gewichtsverlust während neoadjuvanter Therapie soll vermieden werden. Stenosierende Prozesse sollen mechanisch-physikalisch behandelt werden. Die Diskussion um Stent vs. perkutane endoskopische Gastrostomie wird geführt. Patienten erhalten vor und während einer neoadjuvanten Therapie eine diätetische Beratung und falls notwendig eine meist orale Ernährungstherapie. Die präoperative Konditionierung während der neoadjuvanten Therapie eröffnet Patienten die Möglichkeit der Reduktion postoperativer, insbesondere pulmonaler Komplikationen. Die neoadjuvanten Behandlungen sind heute sichere Verfahren. In der aktuellen Literatur existieren nur kasuistische Berichte von Notfallindikationen zur Chirurgie während der neoadjuvanten Therapie. Die endoskopischen Möglichkeiten (Blutstillung bzw. Stent oder Vakuumtherapie) stellen hier die Therapie der ersten Wahl dar. Onkologische Langzeitdaten nach chirurgischer Notfallintervention unter neoadjuvanter Therapie liegen nicht vor.

Der Beitrag von F. Benedix et al. diskutiert das Management perioperativer Komplikationen. Bei der Größe des Operationstraumas dieser Operationen stellt die gezielte präoperative Risikoanalyse und Patientenselektion unter Berücksichtigung des Ernährungszustandes und der Komorbiditäten einen entscheidenden Punkt zur Reduktion von Morbidität und Mortalität dar. Hier ist auch heute die Anastomseninsuffizienz das größte Pro- 
blem. Entscheidend für die erfolgreiche Behandlung sind die frühzeitige Diagnose und der Beginn einer adäquaten Therapie. Jede Verzögerung erhöht das Risiko eines schwerwiegenden, letalen Verlaufs. Anastomoseninsuffizienzen werden in der Mehrzahl durch minimal-invasive endoskopische Techniken wie die Stentimplantation und die endoluminale Vakuumtherapie erfolgreich behandelt. Die Bedeutung der Chirurgie hat im Vergleich zu den endoskopischen Verfahren in den letzten Jahren abgenommen. Eine operative Revision ist bei Vorliegen einer Insuffizienz im frühen postoperativen Verlauf infolge eines technischen Fehlers bei der Anastomosierung erforderlich. Eine weitere Indikation zur chirurgischen Intervention stellt eine fulminante Insuffizienz mit diffuser Peritonitis bzw. Mediastinitis dar. Diese erfordern eine aggressive, zeitnahe Revision mit ausgiebiger Lavage und Drainage, ggf. in Kombination mit einer endoskopischen Intervention. Bei Patienten mit einer ausgedehnten Ischämie bzw. Nekrose des Interponats ist eine Aufhebung der Kontinuität mit Resektion des Interponats, Anlage einer zervikalen Speichelfistel sowie einer enteralen Ernährungssonde unumgänglich. Weiter diskutieren die Autoren die gastrale Entleerungsstörung, welche sich durch eine medikamentöse und endoskopische Intervention gut beherrschen lässt. Eine klare Empfehlung zur Durchführung einer primären Pyloroplastik wird anhand der aktuellen Evidenz nicht abgeleitet. Bei der Behandlung des Chylothorax sollte konservativen Therapiekonzepten der Vorzug gegeben werden. Aufgrund der aktuellen Datenlage ist eine prophylaktische Ligatur des Ductus thoracicus nicht zu empfehlen.

\section{》) Persistierende Fisteln sind operativ zu sanieren}

Der Beitrag von A. Beham et al. widmet sich dem Management von Spätkomplikationen nach Ösophagusresektionen. Treten diese neu auf, besteht oft der Verdacht auf ein Lokalrezidiv. Therapeutisch stehen endoskopische Verfahren im Vordergrund. Bei intakter Passage sind selbstexpandierende Metallstents eine sichere und effektive Methode, wobei die Patienten unter Umständen auch von sog. Antirefluxstents profitieren können. Die intraluminale Hochdosisbrachytherapie ist eine gute Alternative, die unter Umständen einen Überlebensvorteil mit besserer Lebensqualität gewährleistet. Eine tracheoösophageale Fistel sollte primär mit einem Stent in ösophagealer Platzierung versorgt werden. Persistiert die Fistel, ist eine operative Sanierung anzustreben. Der Defekt sollte gegebenenfalls plastisch gedeckt werden. Inwiefern eine Auflösung der Anastomose und eine temporäre kollare Ausleitung des Ösophagus als Speichelfistel sinnvoll ist, sollte vom lokalen Ausmaß der Entzündung und der Mobilität bzw. der Qualität der Durchblutung des hochgezogenen Magenschlauches abhängig gemacht werden. Sollte bei Patienten ohne Pyloroplastik postoperativ eine persistierende Magenentleerungsstörung auftreten, wären neben der prokinetischen Therapie die endoskopische Dilatation des Pylorus und die Injektion von Botox Optionen. Bei Reflux sollte zunächst eine Gastroskopie zum Ausschluss einer mechanischen Ursache sowie einer Einschätzung einer möglichen Entzündung in der Restspeiseröhre durchgeführt werden. Neben funktionellen Störungen des hochgezogenen Magenschlauchs sind narbige Stenosen, vor allem bedingt durch Insuffizienzen der Anastomose, ein Grund für dysphagische Beschwerden. Aufgrund der gestörten Motilität des Magens treten bei etwa $5 \%$ der Patienten milde und bei etwa $1 \%$ schwere Symptome in Sinne eines Dumping-Syndroms auf.

Zusammenfassend kann man feststellen, das endoskopische Verfahren für die Behandlung vieler Komplikationen der chirurgischen Reintervention den Rang abgelaufen haben, da sie deutlich weniger invasiv und damit für den weiteren Verlauf besser sind. Das heißt, Reoperationen werden heute nur in Ausnahmesituationen, die minimal-invasiv endoskopisch nicht zu beherrschen sind, vorgenommen. Eine frühe Diagnostik ist relevant für den Verlauf ebenso das Vorhalten einer kompletten Infrastruktur für das Komplikationsmanagement. Entscheidend für den Behandlungserfolg und die Vermeidung von Komplikationen ist auch bei diesen traumatisierenden Eingriffen sicherlich die präoperative Einschätzung der Operabilität und des Risikos durch eine individuell vorhandene Komorbidität.

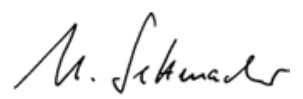

Prof. Dr. Utz Settmacher

\section{Korrespondenzadresse}

\section{Prof. Dr. U. Settmacher}

Klinik für Allgemein-, Viszeral- und

Gefäßchirurgie

Universitätsklinikum Jena

Erlanger Allee 101, Jena

utz.settmacher@med.uni-jena.de

Interessenkonflikt. U. Settmacher gibt an, dass kein Interessenkonflikt besteht. 